\title{
Rocking and Uplift of a Shallow Wind Turbine Foundation
}

\author{
Rose Hawkswood $^{1}$, Helen Mitrani ${ }^{1}$, Mohamed Rouainia ${ }^{1}$, Andrew Brennan ${ }^{2}$ \\ ${ }^{1}$ School of Civil Engineering and Geoscience, Newcastle University \\ Drummond Building, Newcastle upon Tyne, United Kingdom \\ R.m.c.hawkswood1@ncl.ac.uk; Helen.mitrani@ncl.ac.uk; Mohamed.rouaina@ncl.ac.uk \\ ${ }^{2}$ School of Science and Engineering, University of Dundee \\ Fulton Building, Dundee, United Kingdom \\ A.j.brennan@dundee.ac.uk
}

\section{Extended Abstract}

Onshore wind turbine structures are very tall and thin and often have shallow foundations. This type of structure is prone to rocking under applied load [1] and must withstand millions of these load cycles over its design life from the wind and the rotation of the blades [2]. Wind loading is complex and highly variable in frequency and magnitude, with storm events causing large increases of horizontal load. As well rocking, uplift can even occur under these larger design loads.

Previous research has shown that rocking changes the stiffness of the soil below the foundations and therefore the natural frequency of the soil-structure system [2,3]. It has also been shown to cause the soil profile to round below the foundation resulting in a reduction in contact area [4], which can affect the performance of the structure even if the system then ceases to rock. However, rocking can also dissipate energy between the soil and the foundations [3]. This could be beneficial for structures, especially as there is evidence that currently the embedment ring connection at the base of the tower can suffer substantial movement and require remediation [5]. Rocking and uplift behaviour has not been investigated for wind turbine structures and needs to be fully understood to ensure their long-term reliability.

This research uses a combination of numerical and experimental modelling to investigate the behaviour of an onshore wind turbine with shallow foundations, based upon a consolidated clay soil, subject to cyclic loading of varying frequency and magnitude. The loading patterns applied are designed to model normal operation of the turbine interspersed with storm events. This allows investigation of the performance of the structure during and after periods of rocking and uplift.

The experimental modelling is conducted at $50 \mathrm{~g}$ in the $3.5 \mathrm{~m}$ beam geotechnical centrifuge at the University of Dundee. The model is considered to be a 2D scenario, as the cyclic loading is applied in one plane only. Structural settlement and changes in natural frequency of the soil-structure system are measured throughout each test. A container with transparent sides is used to allow the front elevation of the soil and structure to be viewed during each test. This gives information about changes to the soil profile and foundation contact area and whether any uplift is occurring. The addition of markers to the clay enables measurement of soil strains.

The numerical modelling is carried out using a kinematic hardening soil model which is implemented in a finite element procedure and is validated using data from the centrifuge testing. This modelling then extends the investigation by including more realistic wind loading, of increased complexity and number of cycles. 3D models will be also be used to investigate the effect of altering the direction of the wind load and associated rocking.

\section{References}

[1] G. Oliveto, I. Calio, and A. Greco, "Large displacement behaviour of a structural model with foundation uplift under impulsive and earthquake excitations," Earthquake engineering \& structural dynamics, vol. 32, no. 3, pp. 369-393, 2003.

[2] D. Lombardi, S. Bhattacharya, and D. Muir Wood, "Dynamic soil-structure interaction of monopile supported wind turbines in cohesive soil," Soil Dynamics and Earthquake Engineering, vol. 49, pp.165-180, 2013.

[3] S. Gajan, B. L. Kutter, J. D. Phalen, T. C. Hutchinson, and G.R. Martin, "Centrifuge modeling of load-deformation behavior of rocking shallow foundations," Soil Dynamics and Earthquake Engineering, vol. 25, no. 7, pp. 773-783, 2005 . 
[4] R. Paolucci, M. Shirato, and M. T. Yilmaz, "Seismic behaviour of shallow foundations: Shaking table experiments vs numerical modelling," Earthquake Engineering \& Structural Dynamics, vol. 37, no. 4, pp. 577-595, 2008.

[5] M. Currie, M. Saafi, C. Tachtatzis, and F. Quail, "Structural integrity monitoring of onshore wind turbine concrete foundations," Renewable Energy, vol. 83, pp. 1131-1138, 2015 